Journal of Case Reports 2020;10(3):173-176

\title{
Pregnant Woman Infected with Novel Coronavirus
}

\author{
Yulin $\mathrm{He}^{1}$, Yu Zhang ${ }^{2}$, Lihong Xing ${ }^{2}$, Bin $\mathrm{Li}^{1}$, Hongjun $\mathrm{Li}^{3}$, Xiaoping Yin ${ }^{2}$ \\ ${ }^{1}$ Department of Radiology, The First Affiliated Hospital of Nanchang University, Nanchang 330006, China; ${ }^{2}$ Affiliated Hospital of \\ Hebei University, Baoding 071000, China; ${ }^{3}$ Beijing You An Hospital, Capital Medical University, Beijing 100069, China.
}

\section{Corresponding Author:}

Dr Xiaoping Yin

Email: hdfyrad@hbu.edu.cn

This is an Open Access article distributed under the terms of the Creative Commons Attribution License (creativecommons.org/ licenses/by/3.0).

Received : May 31, 2020

Accepted : July 20, 2020

Published : August 25, 2020

\begin{abstract}
Background: Coronavirus disease-19 (COVID-19) is caused by severe acute respiratory syndrome coronavirus 2 (SARS-CoV-2), and is generally susceptible to all people, including pregnant women. Case Report: A pregnant woman was admitted to the hospital with fever. She became covid positive after exposure to her husband who had returned from Wuhan. Her recurring clinical symptoms, chest CT showing sub-pleural patchy shadow resolved following antibiotics and antiviral therapy. Conclusion: Pregnant women are highly sensitive to virus, and her bilateral lung consolidation in the middle and lower field progressed rapidly. When the novel coronavirus nucleic acid or gene test of the patient is negative or turns negative, it is still necessary to combine chest CT examination, and to observe the clinical progress, so as to take relevant treatment measures in time.
\end{abstract}

Keywords: Coronavirus, COVID-19, Pregnancy, Severe Acute Respiratory Syndrome, $\mathrm{X}$-Ray Computed Tomography.

\section{Introduction}

On December 2019, unexplained pneumonia cases appeared in Wuhan. On January 12, 2020, the World Health Organization (WHO) named the pathogenic virus detected as 2019 novel Coronavirus (2019nCoV). On February 7, 2020, the National Health Commission of the Peoples Republic of China named Novel Coronavirus Pneumonia (NCP). At present, WHO renamed the disease as Corona Virus Disease-19 (COVID-19), and International Committee for Taxonomy of Virus (ICTV) named the virus causing it as severe acute respiratory syndrome coronavirus 2 (SARS-CoV-2). Novel coronavirus pneumonia is generally susceptible to all people, including pregnant women. We report a case of a pregnant woman with novel coronavirus infection, and introduce the clinical and imaging findings.

\section{Case Report}

The patient is a 26-year-old woman, 29 weeks pregnant. She was hospitalized with "fever for 3 days". The patient had no contact history of epidemic area. Her husband returned home from Wuhan on January 20, 2020, and her husband had no symptoms. The patient had fever on January 24 , the highest temperature $38.5^{\circ} \mathrm{C}$, with chest tightness in absence of cough. Her initial chest CT showed sub-pleural patchy shadow in the right lung, and the novel coronavirus nucleic acid was positive. She was started on treatment of ceftriaxone, cefoperazone sulbactam and lopinavir. On February 1, 2020, the patient had a fever again, with the maximum temperature of $38^{\circ} \mathrm{C}$, and no obvious discomfort. On February 3, 2020, chest CT showed patches and flake shadows in bilateral lung, most of which were under the pleura. It mainly manifested as consolidation, with air filled 
bronchogram, and ground glass shadow in the middle lobe of the right lung [Fig.1a-c]. Antibiotic and antiviral therapy was continued. Serial laboratory examination is mentioned in Table $\mathbf{1}$. On February 7, 2020 repeat chest CT showed that the consolidation lesions in bilateral lung were obviously absorbed with partial fibrosis [Fig.2a-c]. After treatment, the patient recovered and was discharged on February 8, 2020.

\section{Discussion}

Novel coronavirus pneumonia (COVIP-19) is a disease caused by novel coronavirus, which mainly causes pulmonary inflammatory lesions. The main source of infection is patient infected with novel coronavirus, and asymptomatic infected person may also become the source of infection [1]. Pregnant women are generally susceptible, and more prone to complications [2]. CT plays an important role in the detection of lesions and monitoring of disease changes. When pregnant women receive chest $\mathrm{CT}$ examination, the exposure dose to the fetus is $0.01-0.66 \mathrm{mGy}$ [3]. Therefore, pregnant women suspected of novel coronavirus infection could have a CT chest examination with necessary abdominal protective measures after signing the informed consent [4].

Novel coronavirus pneumonia has strong transmission and high stealthiness. The husband of this patient was from the epidemic area and may be a virus carrier. Chest imaging manifestations of patients with novel coronavirus pneumonia are characterized in the early stage by multiple ground glass density shadows under the pleura and thickened blood vessels and thick-walled bronchus can be seen passing through. The patient's initial chest CT showed the changes of sub-pleural ground glass in the lower lobe of the right lung. The clinical symptoms reappeared, but the coronavirus nucleic acid test was negative repeatedly. All the absolute value of lymphocyte subgroup were decreased during this period. Chest CT reexamination later showed that the range of

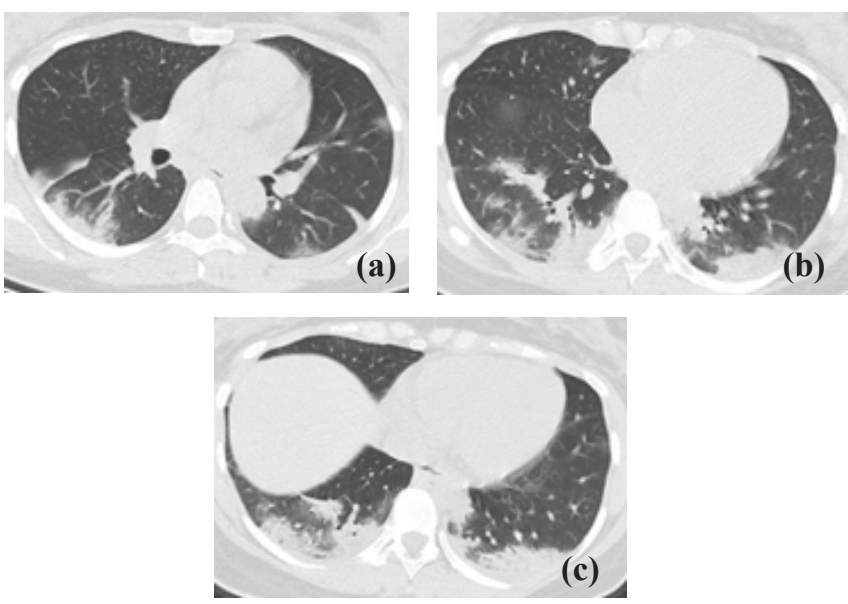

Fig.1(a-c): The patches and flake shadows in bilateral lung, most of which were under the pleura, and mainly manifested as consolidation, with air filled bronchogram, and ground glass shadow in the middle lobe of the right lung.

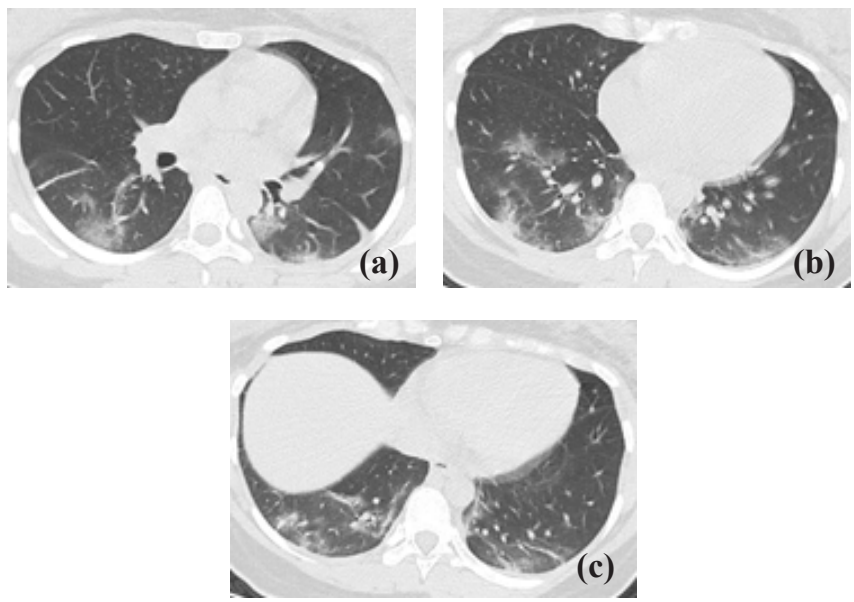

Fig.2(a-c): The consolidation lesions in bilateral lung were absorbed with partial fibrosis.

the lesions was enlarged, and consolidation could be seen, and the lesions changed rapidly, which was considered as progression [5-7]. Subsequent blood routine examination showed that the white cells and lymphocytes were lower than before, which proved disease progression.

In the general population, the progression imaging of novel coronavirus pneumonia mostly shows that the ground glass density shadow progresses from periphery to center. But due to immune and endocrine changes during pregnancy, 
Table 1: Laboratory Values during the Course of Illness.

\begin{tabular}{|c|c|c|c|c|c|c|c|c|}
\hline \multicolumn{3}{|l|}{ Variable } & \multicolumn{6}{|c|}{ Date of Laboratory Examination (2020) } \\
\hline Laboratory Values & $01-24$ & $01-27$ & $01-29$ & $02-31$ & $02-01$ & $02-02$ & $02-05$ & $02-07$ \\
\hline 2019 novel coronavirus ORFlab & $(+)$ & & $(-)$ & $(-)$ & & $(-)$ & & $(-)$ \\
\hline White cells $\left(\times 10^{9} / \mathrm{L}\right)$ & & 4.5 & & & & & 2.87 & 4.19 \\
\hline Red cells $\left(\times 10^{9} / \mathrm{L}\right)$ & & 2.72 & & & & & 2.85 & 2.81 \\
\hline Absolute value of lymphocytes $\left(\times 10^{9} / \mathrm{L}\right)$ & & 0.74 & & & & & 0.14 & 1.49 \\
\hline Erythrocyte sedimentation rate $(\mathrm{mm} / \mathrm{h})$ & & 30 & & & & & 100 & \\
\hline Procalcitonin (ng/mL) & & $<0.25$ & & & & & 0.10 & \\
\hline Respiratory pathogen nucleic acid test 13 & & $(-)$ & & & & & & \\
\hline Absolute value of CD3+CD8+ cells $(/ \mu \mathrm{L})$ & & & & 212 & & & & \\
\hline Ratio of CD3+CD8+ cells (\%) & & & & 36.1 & & & & \\
\hline Ratio of CD4/CD8 & & & & 0.83 & & & & \\
\hline Absolute value of CD56+CD16+CD3- cells $(/ \mu \mathrm{L})$ & & & & 34 & & & & \\
\hline Ratio of CD56+CD16+CD3- cells (\%) & & & & 5.8 & & & & \\
\hline Absolute value of CD19+CD3- cells $(/ \mu \mathrm{L})$ & & & & 53 & & & & \\
\hline Ratio of CD19+CD3- cells \% & & & & 9.0 & & & & \\
\hline IL-6 (pg/mL) & & & & & 22.70 & & & \\
\hline
\end{tabular}

pregnant women are highly sensitive to virus. The lesions progressed rapidly, mainly consolidation, mostly in the middle and lower field of bilateral lung $[8,9]$. The longer the time interval between chest CT examination and onset of clinical symptoms, the more obvious the abnormal image of the lung, there are more consolidation lesions than before and consolidation can be used as a marker of disease progression [10]. After further treatment, the blood routine examination showed that the white cells and lymphocytes returned to normal. Chest CT for reexamination showed that the ground glass and consolidation lesions were obviously absorbed, the density becoming light, and that residual fiber strip shadows were seen. These signs suggested recovery. The CT manifestation of the novel coronavirus pneumonia recovery is similar between pregnant women and the general population.
At present, there are few reports about novel coronavirus infection in pregnant women. According to the epidemiological history of this case, combined with clinical, imaging data and positive nucleic acid test, the disease was confirmed.

\section{Conclusion}

Viral nucleic acid detection has strong specificity but poor sensitivity. When typical clinical manifestations and characteristic imaging manifestations of lung appear in the environment of clustering disease, it should be suspected diagnosis, whether the novel coronavirus nucleic acid or gene detection is positive or not [1]. Isolation treatment is needed to reduce the possibility of infecting others. Pregnant women are highly sensitive to virus, and the lesion progresses rapidly. In the process of diagnosis and treatment, CT plays 
an important role in detecting the progress and outcome of the disease. It can reduce and avoid the misdiagnosis of the patients whose clinical and imaging manifestations are inconsistent to take relevant measures in time.

Contributors: Yulin HE and Yu ZHANG were responsible for the writing the manuscript and the conception of the work; Lihong XING, Bin LI, Hongjun LI and Xiaoping YIN were responsible for revising it critically. Yulin HE will act as a study guarantor. All authors approved the final version of this manuscript and are responsible for all aspects of this study.

Funding: None; Competing interests: None stated.

\section{References}

1. National Health Commission of the Peoples Republic of China, National Administration of Traditional Chinese Medicine, Guidelines on diagnosis and treatment of novel coronavirus pneumonia (Trial sixth edition). Chin Infect Control. 2020;19:192-195.

2. Wong SF, Chow KM, Leung TN, Ng WF, Ng TK, Shek $\mathrm{CC}$, et al. Pregnancy and perinatal outcomes of women with severe acute respiratory syndrome. Am J Obstet Gynecol. 2004;191:292-297.

3. Tremblay E, Thérasse E, Thomassin-Naggara I, Trop I. Quality initiatives: guidelines for use of medical imaging during pregnancy and lactation. Radiographics. 2012;32:897-911.

4. Dunjin Chen, Huixia Yang. Expert advice on novel coronavirus infection in pregnancy and puerperium [EB/ OL]. Chinese Society of Perinatal medicine, Available at: http://www.cspm.org.cn/comsite/news/show/cn/3098. html, 2020-02-05. Accessed on March 31, 2020.

5. Committee of the Infectious Diseases Radiology Group of Chinese Society of Radiology; Committee of the Infectious Diseases Radiology Section of Chinese Medical Doctor Association; Infectious Disease Imaging Group, Infectious Disease Branch, Chinese Research Hospital Association; Imaging Committee of Chinese Association for the Prevention and Treatment of STD AIDS Infection (Infectious Disease); Infectious Diseases Group, General Radiological Equipment Committee, China Association of Medical Equipment; Beijing Imaging Diagnosis and Treatment Technology Innovation Alliance. Guideline for imaging diagnosis of novel coronavirus $(2019-\mathrm{nCoV})$ infected pneumonia $\left(1^{\text {st }}\right.$ edition 2020). New Medicine. 2020;30:22-34.

6. Lei J, Li J, Li X, Qi X. CT imaging of the 2019 novel coronavirus (2019-nCoV) pneumonia. Radiology. 2020;295:18.

7. Guan H, Xiong Y, Shen N, Fan Y, Shao J, Li H, et al. Clinical and thin-section CT features of patients with 2019-nCoV-pneumoni. Radiologic Practice. 2020:1-6.

8. Zhu W, Liu Y, Lu P, Li, G Zhou J, Zhou J, et al. Thoracic imaging characteristics in pregnant/parturient women with influenza A (H1N1) pneumonia. Radiologic Practice. 2010;25.

9. Koo H J, Lim S, Choe J, Choi SH, Sung H, Do KH. Radiographic and CT features of viral pneumonia. Radiographics. 2018;38:719-739.

10. Song F, Shi N,Shan F, Zhang Z, Shen J, Lu H, et al. Emerging coronavirus 2019-nCoV pneumonia. Radiology. 2020;295:210-217. 